\title{
COMPUTED TOMOGRAPHY OF CRYOGENIC CELLS
}

\author{
G. SCHNEIDER, and E. ANDERSON \\ Center for X-ray Optics, Lawrence Berkeley National Laboratory, \\ One Cyclotron Road MS 2-400, \\ Berkeley, CA 94720, USA \\ S. VOGT, C. KNÖCHEL, and D. WEISS \\ Institut für Röntgenphysik, Universität Göttingen, Geiststraße 11 \\ D-37073 Göttingen, Germany \\ M. LEGROS, and C. LARABELL \\ Life Sciences, Lawrence Berkeley National Laboratory, \\ One Cyclotron Road MS 6-2100, \\ Berkeley, CA 94720, USA \\ Received (to be inserted \\ Revised by publisher)
}

\begin{abstract}
Soft X-ray microcopy has resolved $30 \mathrm{~nm}$ structures in biological cells. To protect the cells from radiation damage caused by X-rays, imaging of the samples has to be performed at cryogenic temperatures, which makes it possible to take multiple images of a single cell. Due to the small numerical aperture of zone plates, X-ray objectives have a depth of focus on the order of several microns. By treating the X-ray microscopic images as projections of the sample absorption, computed tomography (CT) can be performed. Since cryogenic biological samples are resistant to radiation damage, it is possible to reconstruct frozen-hydrated cells imaged with a full-field X-ray microscope. This approach is used to obtain three-dimensional information about the location of specific proteins in cells. To localize proteins in cells, immunolabelling with strongly X-ray absorbing nanoparticles was performed. With the new tomography setup developed for the X-ray microscope XM-1 installed at the ALS, we have performed tomography of immunolabelled frozen-hydrated cells to detect protein distributions inside of cells. As a first example, the distribution of the nuclear protein, male specific lethal 1 (MSL-1) in the Drosophila melanogaster cell was studied.
\end{abstract}

\section{Introduction}

The structure of proteins can be studied by X-ray crystallography with atomic resolution, but their location in cells remains unknown. With immunolabelling it is possible to localize these proteins in cells. Up to now light microscopy has mainly been used to study their distribution in cells by tagging the investigated protein with fluorophore-conjugated antibodies. While light microscopes allows routine investigation of whole, unsectioned cells, the obtainable resolution is diffraction limited to about $200 \mathrm{~nm}$. In addition, this technique reveals mainly the distribution of the fluorophore-conjugated antibodies whereas most unlabelled cell structure is not clearly visualized.

Electron microscopy can reveal cell structures at much higher resolution level, but is limited by the thickness of the sample, i.e. only less than $1 \mu \mathrm{m}$ thick objects can be imaged. Therefore, no conventional imaging technique exists which can visualize the threedimensional distribution of proteins inside whole hydrated cells, e.g. in the cell nucleus, with higher than light microscopical resolution. Due to the shorter wavelengths of X-rays than visible light, X-ray microscopy provides higher resolving power than light microscopes. By utilizing the natural absorption contrast between protein and water at photon energies of about $0.5 \mathrm{keV}$, smallest cell structures of about $30 \mathrm{~nm}$ in size embedded in vitreous ice can be detected in X-ray microscope images ${ }^{1-3}$. The aim of this work is to apply computed tomography, which has already been demonstrated using artificial samples ${ }^{4}$, mineralized sheats of bacteria ${ }^{5}$ and frozen-hydrated algae ${ }^{6}$, in order to localize specific proteins and organelles in unsectioned, frozen-hydrated cells.

\section{Lateral Resolution and Depth of Focus}

The computed-tomography experiments presented in this work are all based on tilt series of images acquired using the amplitude contrast mode of the TXM. In the amplitude contrast mode, the microscope forms enlarged images of the intrinsic photoelectric absorption contrast of the object. However, the obtained image contrast is influenced both by the condenser illuminating the object and by the imaging X-ray objective. The e-beam written condenser zone plate used for these experiments has an outermost zone width of $\mathrm{dr}_{\mathrm{N}}=54 \mathrm{~nm}^{7}$. At $2.4 \mathrm{~nm}$ wavelength, the numerical aperture is given by $\mathrm{NA}_{\text {cond }}=\lambda /\left(2 \mathrm{dr}_{\mathrm{N}}\right)=0.0222$. 
Correspondingly, the numerical aperture of the X-ray objective micro zone plate with an outermost zone width of $40 \mathrm{~nm}$ is $\mathrm{NA}_{\mathrm{obj}}=0.03$. Note that while the imaging micro zone plate has a solid or full-cone aperture, the condenser zone plate has a central stop to block the undiffracted radiation from the field of view. The illuminating aperture is therefore shaped like a hollow cone. The presence of a shadow aperture influences the modulation transfer to some degree ${ }^{1}$; this effect is neglected in the following considerations. The ratio of the apertures is called the degree of partial coherence $\mathrm{m}$; in this case, $\mathrm{m}=\mathrm{NA}_{\text {cond }} / \mathrm{NA}_{\mathrm{obj}}=0.74$. In order to be able to employ the useful properties of linear systems to calculate the lateral resolution and the depth of focus of the microscope, it is desirable to model the image formation as either coherent or incoherent, even if neither of these extreme cases is exactly given. For the experiments described in this work, this means approximating $\mathrm{m}=0.74$ either as $\mathrm{m}=$ 0 or as $m=1$. Hopkins and Barham calculated the influence of the relative condenser aperture on the Rayleigh resolution of two pinholes, showing that by reducing the degree of partial coherence from $\mathrm{m}=1$ to $\mathrm{m}=0.74$, the Rayleigh resolution is reduced by only $8 \%$. Therefore, the image formation in the TXM is assumed to be in good approximation incoherent, and can be described as a linear system acting on the intensity in the object plane. This means that the intensity in the image plane $I_{i m}$ is given by a convolution of the intensity in the object plane $\mathrm{I}_{\mathrm{ob}}$ with the point spread function (PSF) $\mathrm{I}_{\mathrm{PSF}}$.

If the micro zone plate objective is illuminated with monochromatic radiation and used in one selected focusing diffraction order, the optical performance is that of a thin lens. In particular, the zone plate PSF for incoherent image formation is given by the intensity distribution in the vicinity of the geometric focal point of a thin lens. This intensity $\mathrm{I}_{\mathrm{PSF}}$ can be calculated by approximating the Huygens-Fresnel integral for mono-

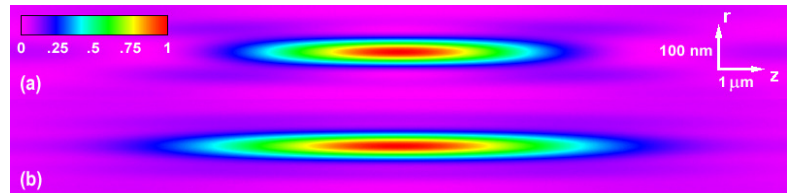

Fig. 1. The normalized point spread function $\mathrm{I}_{\mathrm{PSF}} / \mathrm{I}_{0}$ (intensity near the geometric focal point) of a zone plate objective as a function of radius $r$ and of defocus $z$ for (a) monochromatic radiation of $2.4 \mathrm{~nm}$ wavelength and (b) narrow-bandwidth radiation with a mean wavelength $\lambda_{0}=2.4 \mathrm{~nm}$ and a monochromaticity $\lambda_{0} / \Delta \lambda=200$, for a zone plate with an outermost zone width ${d r_{N}}=40 \mathrm{~nm}$ and a numerical aperture NA $=0.036$. The $\mathrm{z}$-axis has been condensed by a factor of 10 . Note that the narrow-bandwidth PSF is more elongated than the monochromatic PSF, indicating a greater depth of focus. In the focal plane, the secondary maxima are more pronounced in (b) than in (a), indicating decreased lateral resolution for narrow-bandwidth radiation.

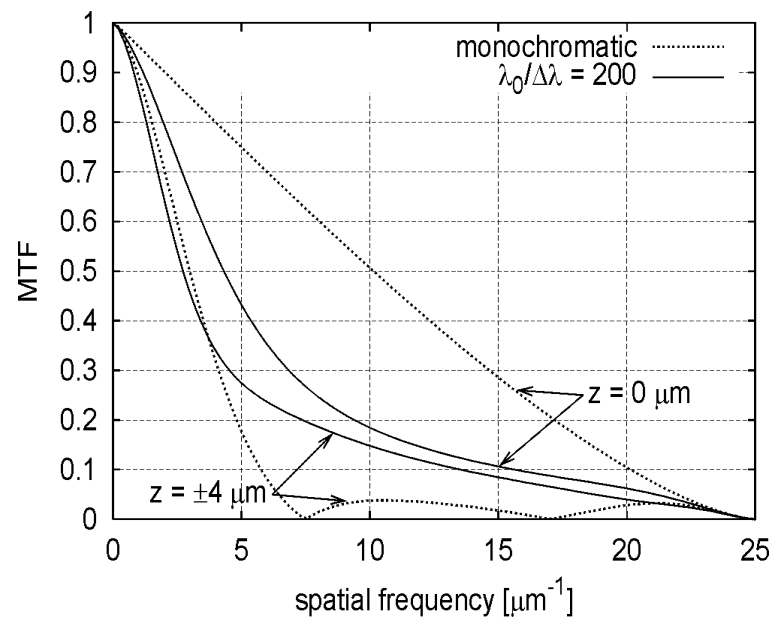

Fig. 2. The modulation transfer function (MTF) as a function of spatial frequency for different values of the defocus $\mathrm{z}$ (zone plate parameters as in Figure 1$)^{6}$. The object mainly used in the tomography experiments has a diameter of approx. $8 \mu \mathrm{m}$, the maximum defocus for any object part is therefore $\mathrm{z}= \pm 4$ $\mu \mathrm{m}$. For this defocus, the narrow-bandwidth MTF is more favorable than the monochromatic MTF.

chromatic images of a point source by a circular aperture. In the TXM, a combination of a condenser zone plate and a pinhole is used to monochromatize the polychromatic synchrotron radiation and to illuminate the object. The spectral distribution of the illuminating radiation is approximately Gaussian in shape, characterized by a mean wavelength $\lambda_{0}$ and a full width at half maximum of $\Delta \lambda$. Raytracing calculations yield that the monochromaticity of the illumination is $\lambda_{0} / \Delta \lambda=200$. Fresnel zone plates are chromatic lenses, i.e., the focal length is a function of wavelength. To calculate the effective point spread function for this kind of narrow-bandwidth illumination, the monochromatic PSF was computed for a large number of (equidistantly spaced) wavelengths, and the different monochromatic PSFs were added up. Image formation is thus again modeled as being completely incoherent, with the contributions from different illuminating wavelengths adding up to form the total image intensity. As shown in Figure 1, adding up the monochromatic PSFs therefore results in an elongation of the PSF $^{5}$.

The modulus of the Fourier transform of the PSF is called modulation transfer function (MTF), and describes the contrast transfer from object to image intensity as a function of the spatial frequency. In order to characterize the imaging properties of the X-ray microscope, MTFs were calculated for different object planes both for monochromatic radiation and for the narrow-bandwidth radiation (see Figure 2). The MTF for the focused plane $(\mathrm{z}=0)$ and monochromatic radiation has a form which is well known for incoherent image formation. Only object structures with the spatial frequency $\mathrm{k}=0$ show a modulation transfer of 1 , 
meaning that the integral intensity is preserved under imaging. Object structures with higher spatial frequencies have reduced modulation transfer. The cutoff frequency of $25 \mu^{-1}$ corresponds to a periodicity of $40 \mathrm{~nm}$, i.e., the outermost zone width of the imaging micro zone plate objective. The periodicity of the Rayleigh resolution, $1.22 \mathrm{dr}_{\mathrm{N}}$, corresponds to a spatial frequency of $20.5 \mu^{-1}$. As can be seen in Figure 2, at this frequency there is a modulation transfer of approx. $9 \%$. For narrow-bandwidth radiation with $\lambda_{0} / \Delta \lambda=200$, the modulation transfer in the focal plane is considerably worse than for monochromatic radiation, both for high and low spatial frequencies. For example, the $9 \%$ modulation transfer corresponding to the Rayleigh resolution occurs at $16.7 \mu^{-1}$, with a resolution equivalent of $60 \mathrm{~nm}$.

The diameter of the Drosophila melanogaster cell studied in this work is approx. $8 \mu \mathrm{m}$. If the focused plane is adjusted to go through the center of the object, this means that the maximum defocus for any part of the object is $\mathrm{z}= \pm 4 \mu \mathrm{m}$. It is therefore necessary to compare the MTF for a defocus of $z= \pm 4 \mu \mathrm{m}$ to that of the focal plane at $\mathrm{z}=0$. For the narrow-bandwidth MTF, there is little difference between the focused and the defocused plane, while the monochromatic MTF shows substantially different modulation transfer at $\mathrm{z}= \pm 4 \mu \mathrm{m}$.

In summary, the narrow-bandwidth radiation generated by the linear monochromator decreases the lateral resolution in the focused object plane compared to that of monochromatic radiation. At the same time it significantly increases the depth of focus, so that for an object with a diameter of $8 \mu \mathrm{m}$, the modulation transfer function is approximately the same for the whole object. In the case of a geometric projection, the image contrast is equal to the photoelectric absorption contrast of the object for all spatial frequencies. In our case of $\mathrm{X}$-ray microscopic images, the image contrast is the result of a linear transformation of the photoelectric absorption contrast. The main difference between geometric projections and X-ray microscopic images acquired with narrow-bandwidth illumination is that high spatial frequencies are attenuated in the microscopic images, however nearly equally for all parts of the object. This effect is neglected in the tomographic reconstructions presented in this work.

\section{Experiment}

\subsection{MSL-1 Protein in Drosphila melanogaster}

Proteins carry out specific functions in cells, often at discrete locations. Therefore, mapping their distribution is important to understand their biological function. For example, nuclear proteins regulate the dosage compensation, an essential biological process in many species. This process equalizes transcription of $\mathrm{X}$ linked genes in females and males. In Drosophila melanogaster this is accomplished by several proteins forming a complex on the $\mathrm{X}$ chromosome, and enhancing X-linked transcription. In X-ray microscopic images of cells specific proteins cannot be identified directly inside a cell. This can only be accomplished by tagging these proteins with markers, e.g., using immunolabelling procedures. The distribution of one of these proteins, male-specific-lethal-1 (MSL-1), in the cell nuclei of male Drosophila cells were mapped using $\mathrm{X}$-ray and confocal laser scan microscopy. Figure 3 shows the confocal laser scan micrograph on the left, the brightly fluorescing areas respresent the label ${ }^{2}$. On the right, the same cell with the same orientation is shown in an X-ray micrograph. Here, the labelled structures appear dark, due to the strong X-ray absorption of the silver enhanced gold spheres. In both micrographs, the same regions appear labelled. A spatial frequency analysis was performed on the image data. Smallest visible structures in the X-ray micrograph are about $30 \mathrm{~nm}$, in the confocal micrograph about $180 \mathrm{~nm}$ in size. To get also high spatial resolution information on the 3D configuration of the cell and the labelled regions, we performed tomography of frozenhydrated Drosophila melano-gaster cells. The setup for the tomography experiment and first results are presented below.

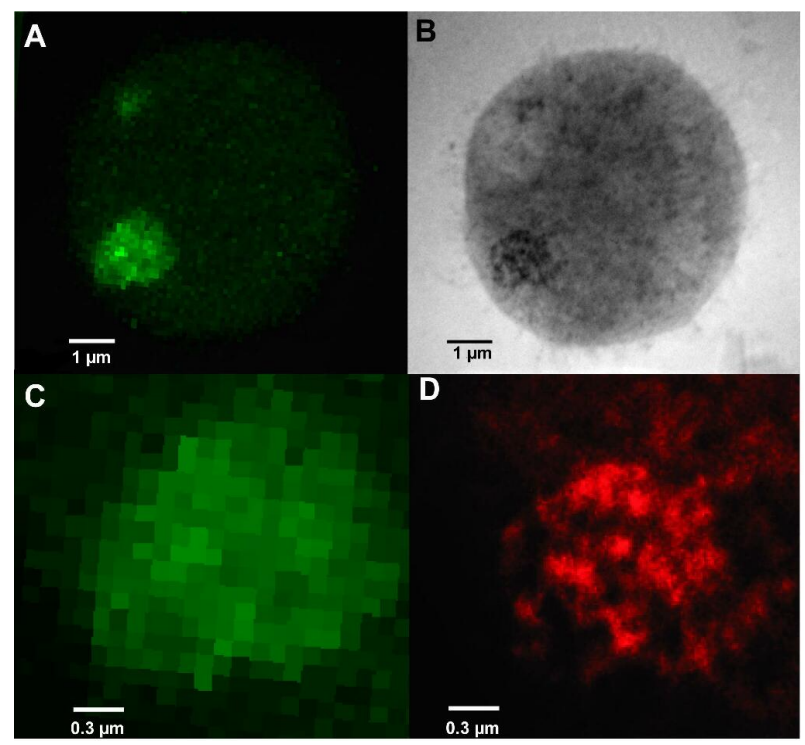

Fig. 3. Micrographs of the same MSL-1-labelled cell in the confocal light and in the cryo-X-ray microscope ${ }^{2}$. The locations of the labelled structures are identical in both micrographs. The substructures of the labelled regions appear much more detailed in the TXM (B) than in the LSM micrograph (A); (C) shows a magnified region of the LSM micrograph (A); for a better visualization the same region from the TXM micrograph is also shown magnified in false colors in (D). The scaling was inverted, so that X-ray-dense structures (e.g., labelled parts) appear brighter. 


\subsection{Experimental Setup}

In order to obtain a three-dimensional image of an Xray microscopic specimen using computed tomography, the specimen must be rotated around an axis perpendicular to the microscope optical axis, and imaged under a large range of viewing angles. To avoid reconstruction artifacts caused by limited projection angles, it is highly desirable to employ a rotationally symmetric holder permitting image acquisition over the full angular range of $180^{\circ}$. Such a geometry is provided by object holders made of glass capillaries. These holders for tomographic X-ray microscopy experiments were first used in the cryo TXM operating at the BESSY electron storage ring ${ }^{6}$. Figure 4 shows light microscopical images of such a glass capillary. Due to the thin wall of the glass capillary, X-rays of $520 \mathrm{keV}$ photon energy penetrate such a glass capillary which is filled with water and a biological sample. Under these conditions the average transmission is about $15 \%$. To protect biological cells from radiation damage, they have to be imaged at cryogenic temperatures at about $-150^{\circ} \mathrm{C}$. At the resolution level of about $30 \mathrm{~nm}$, it was demonstrated that biological cells in vitreous ice can tolerate a radiation dose about a 100 times higher than the dose limit observed in cryo TEM ${ }^{1}$.

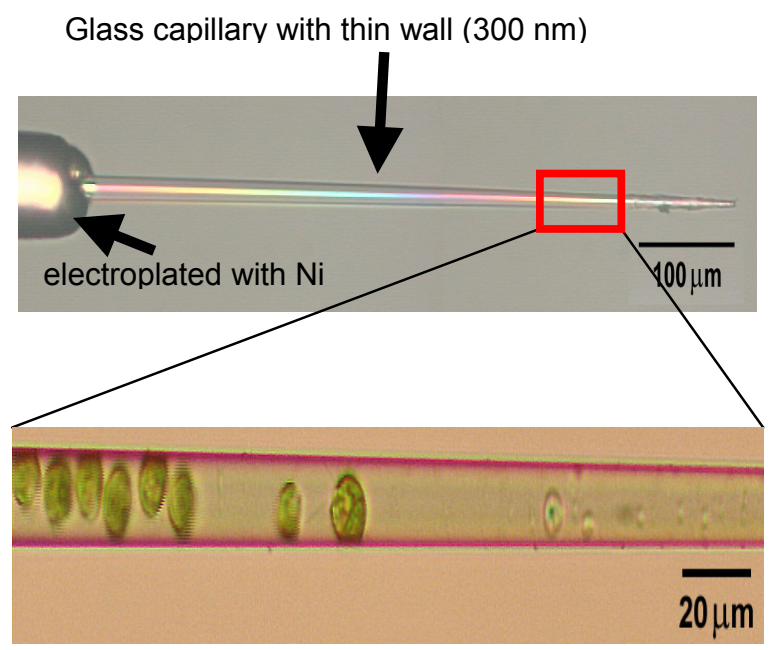

Capillary with $10 \mu \mathrm{m}$ in diameter

Fig. 4. Reinforced capillary holder for computed-tomography experiments at cryogenic temperatures. To ensure the mechanical holder stability under cryo conditions, the tapering tip of the borosilicate glass capillary is reinforced with an electroplated, $25 \mu \mathrm{m}$ thick nickel coat (left image edge), leaving only the uppermost $500-800 \mu \mathrm{m}$ of the tip Xray transparent. The nickel coat prevents oscillations of the capillary in the cryogenic He-gas. Prior to electroplating, the tip was sealed by dipping it into silicone rubber. This (empty) tip is still sealed with a drop of silicone rubber; the foremost $100 \mu \mathrm{m}$ are coated with the rubber and must be removed to make the holder usable. Note the changing capillary wall thickness (decreasing from left to right) indicated by the interference colors.

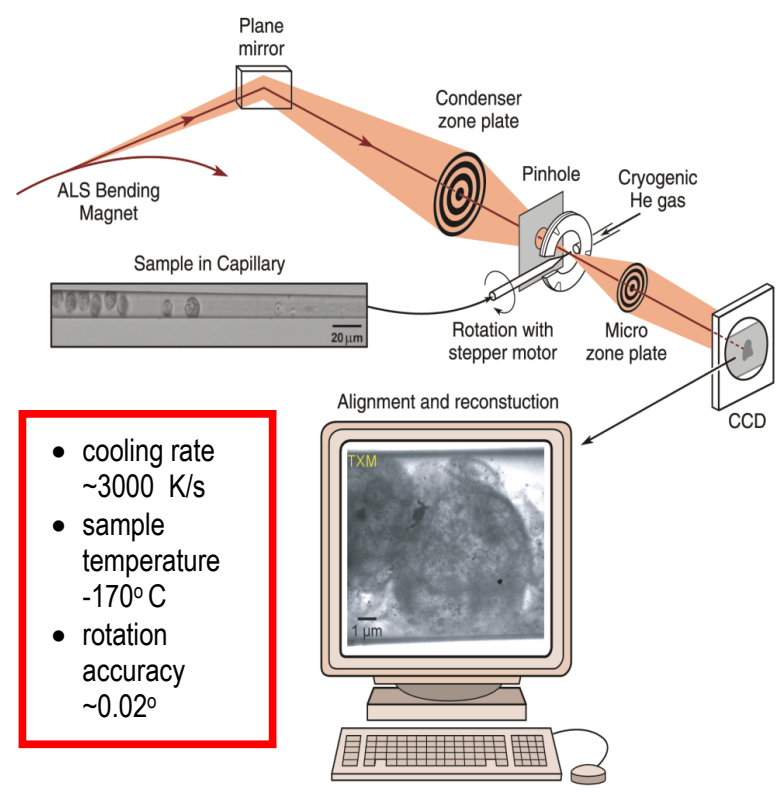

Fig. 5. Schematic diagram of the capillary sample holder based cryo tomography experiment performed using a soft $\mathrm{X}$-ray transmission microscope, XM-1 at the ALS in Berkeley. A condenser zone plate lens (KZP) illuminates the sample with soft $\mathrm{X}$ - rays from a bending magnet $\mathrm{X}$-ray source with $520 \mathrm{eV}$ photon energy. An objective zone plate (MZP) with $40 \mathrm{~nm}$ smallest zone structures generates a $2000 \times$ magnified image onto a back-thinned CCD camera. Under these imaging conditions, the depth of field of the XM-1 imaging system is close to $8 \mu \mathrm{m}$ without significantly reduced lateral resolution. Under these conditions the magnified images are in good approximation projections of the specimens X-ray transmission. By rotating the capillary through $180^{\circ}$ in $4^{\circ}$ angular steps, and recording the transmission image data at each orientation, the 3-D linear absorption coefficient has been reconstructed using multiplicative algebraic reconstruction techniques (MART).

In the cryo TXM operating at BESSY, the capillaries were handled inside an environment filled with cryogenic nitrogen gas. The TXM operating at the ALS electron storage ring was designed to image samples at room temperature, therefore, a new tomographic setup was developed. The setup of the cryo transmission Xray microscope at the ALS is shown in Figure 5 (see also Figure 6 showing a photograph of the object stage). In this cryo TXM liquid nitrogen cooled He gas is used to shock-freeze the sample in the capillary and to maintain it at low temperatures during data acquisition. To avoid vibrations and drift of such a thin sample holder in the cryo TXM, the capillaries are electroplated with nickel, coating all but the final 500 $800 \mu \mathrm{m}$ which can be used for imaging samples (see Figure 4). 


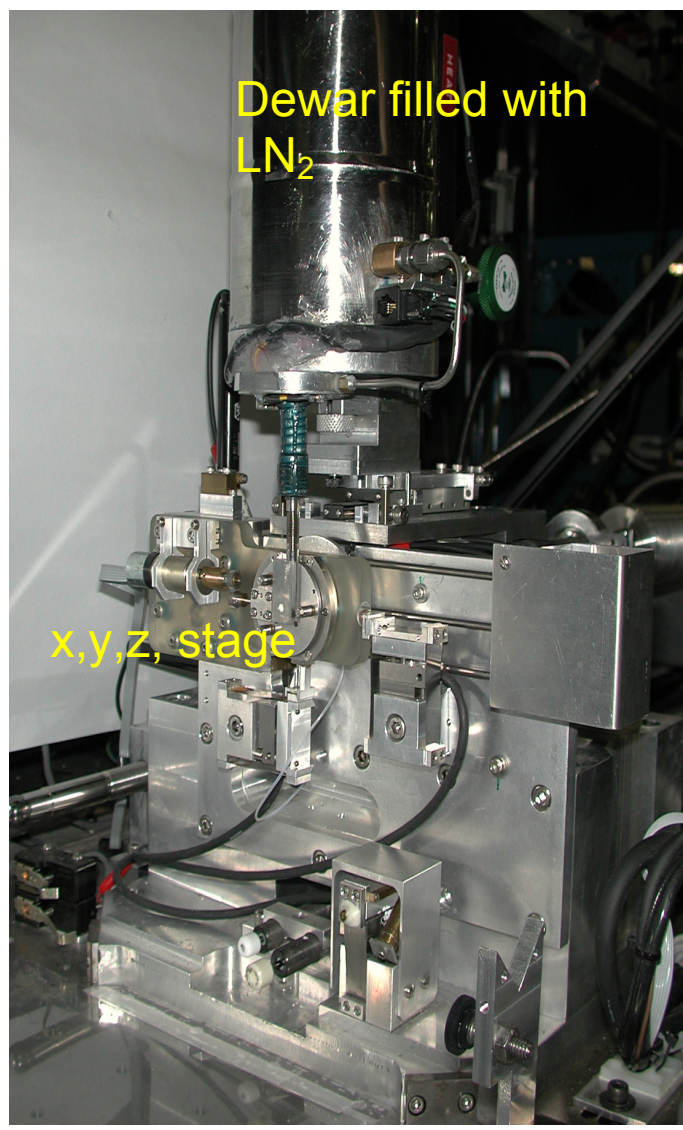

\section{Object stage of the Cryo $\mathrm{X}$-ray Microscope, $\mathrm{XM}-1$}

\section{Rotation stage for capillaries}

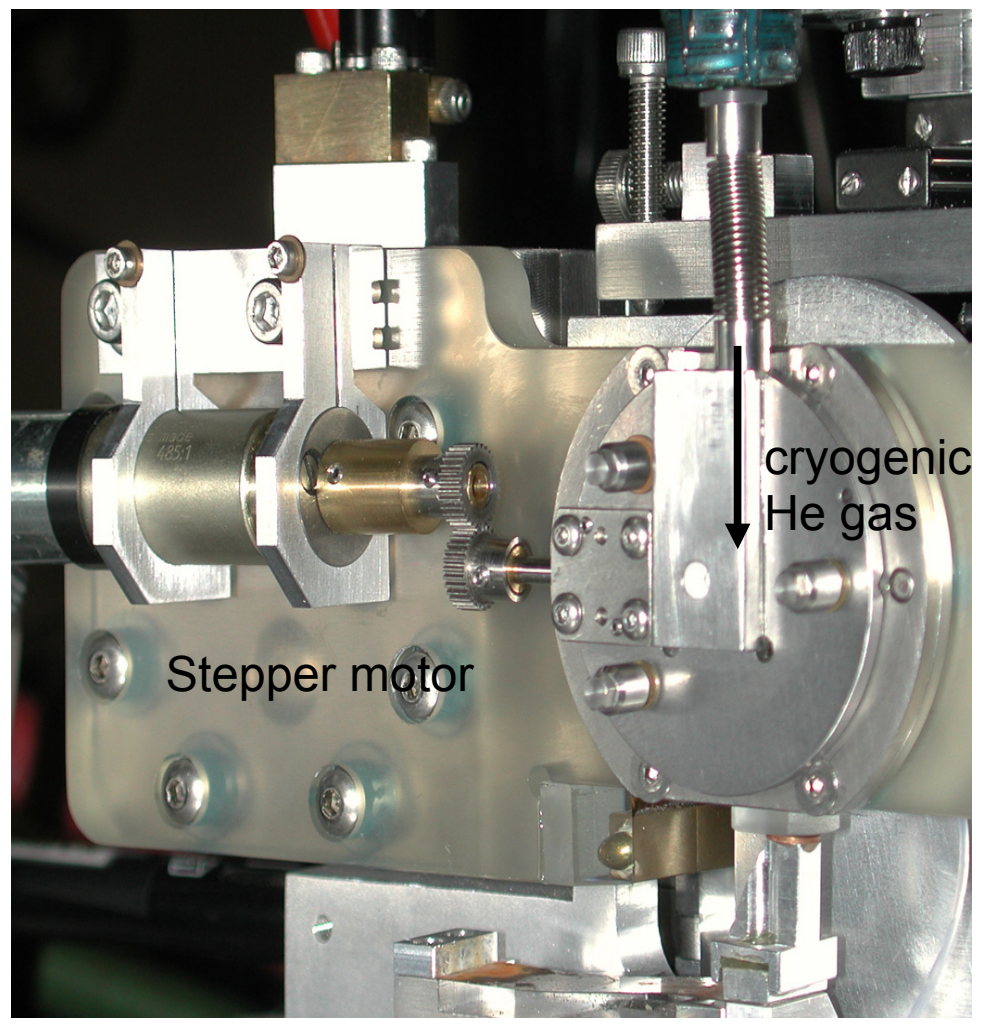

Fig. 6. Photograph of the object stage with the tomographic setup for the capillaries. The $\mathrm{He}$ gas is cooled by liquid nitrogen inside the dewar and then directed onto the capillary to keep it at cryogenic temperatures. The axis of the generated He jet and the capillary axis have to be parallel to avoid sample vibrations which were observed when adjusting both axis perpendicular to each other.

\section{Computed Tomography of Labelled Drosophila Melanogaster Cells}

To visualize the MSL-1 protein in the cell nuclei, the cells were labelled with a $1 \mathrm{~nm}$ colloidal gold conjugated antibody and then silver enhanced to increase the absorption contrast of the label. After filling the cell suspension into the capillaries, they were imaged at $520 \mathrm{eV}$ photon energy with a nickel zone plate objective with $40 \mathrm{~nm}$ outermost zone width. Figure 7 shows the first of the 48 different viewing angles spanning an angular range of $180^{\circ}$. This tomographic data set was collected in 3 hours. During aquisition of the tilt series, the specimen was held at cryogenic temperatures. In order to align all the different viewing angles to a common rotation axis, we added colloidal gold particles with $100 \mathrm{~nm}$ in diameter as fiducial markers. The image alignment was performed using an algorithm developed for electron tomography ${ }^{9}$. Using this 3D marker model, the alignment can be done very precisely with about $10 \mathrm{~nm}$ accuracy, because only the center of the gold particles has to be determined. After alignment, a multiplicative algebraic reconstruction technique (MART) was applied to reconstruct the three-dimensional structure of the cells inside the capillary. Figure 7 shows different $150 \mathrm{~nm}$ thick slices perpendicular through the capillary axis. The internal structure of the cells as well as the label and the added gold particles are visible.

Some conclusions can be drawn from the obtained data set. As expected, MSL-1 is not present in the nucleoli of the cells. Instead, part of the MSL-1 labelled region appears to lie in close spatial relation to the nucleolus. However, the questions whether there is indeed a spatial relation, possibly cell cycle dependent, between X-chromosome and nucleolus which causes part of the MSL-1 labelled area to lie near the nucleolus of a cell cannot be solved by this data set. Further studies are necessary to acquire tomographic data sets 

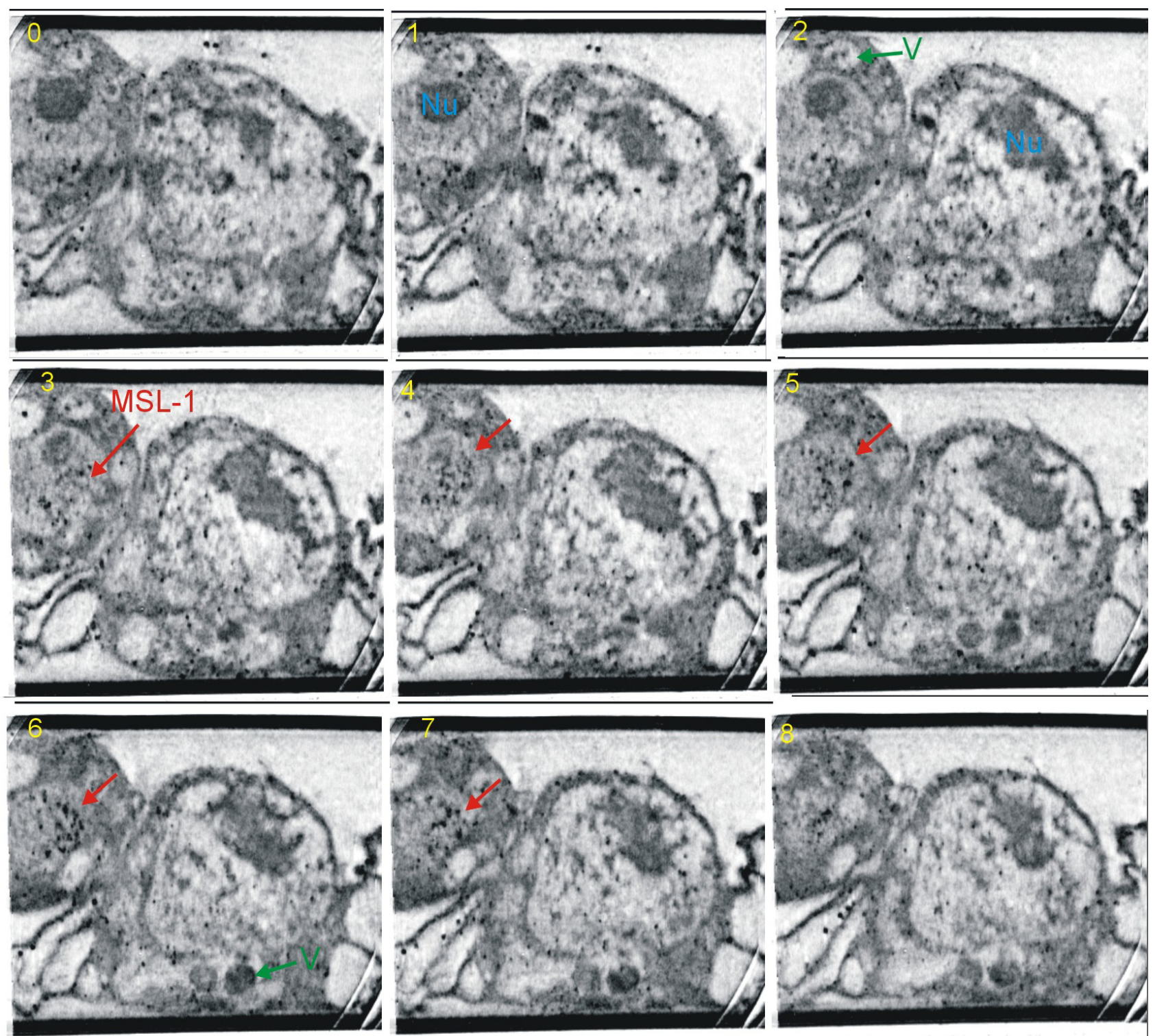

Fig. 7. TXM micrograph (right) showing the first of the 48 X-ray microscope images of immunogold labelled Drosophila melanogaster cells constituting the tilt series. Panels (0) - (8) show parallel slices of the reconstructed local linear absorption coefficient. The capillary axis is parallel to the plane of the paper. Each slice is $150 \mathrm{~nm}$ thick. Low absorption maps to light gray, and high absorption to dark gray. The distribution of the labelled nuclear protein inside the cell nuclei is visualized in the slices by the strong X-ray absorbing silver enhanced gold particles. Details down to $60 \mathrm{~nm}$ size inside the cells can be detected (e.g., vesicles (V) and nucleolus $(\mathrm{Nu}))$.

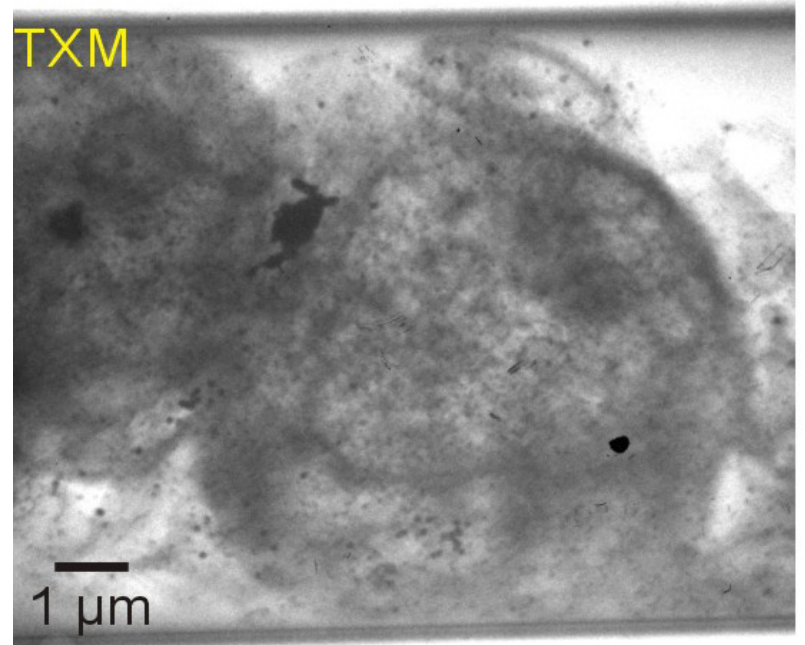


of sufficient numbers of cells to be able to draw statistically relevant conclusions.

In comparison with two-dimensional X-ray micrographs of these cells (see Figure 7, lower right), it is clearly demonstrated that tomographic reconstructions of labelled cells show much more cell details than can be discerned in the $2 \mathrm{D}$ images. Not only is the three-dimensional arrangement of the label with respect to the three-dimensional structure of the cell visualized, but it is possible to detect labelled areas with a higher sensitivity. To achieve the best possible results, it is furthermore necessary to reduce the artifacts introduced into the specimen by the chemical treatment of the cells for immunolabelling. This can be done by in vivo immunolabelling.

\section{Summary}

Due to the short wavelengths of X-rays and low numerical aperture of the Fresnel zone plates used as Xray objectives, the depth of field is several microns. Within the focal depth, imaging a thick specimen is to a good approximation equivalent to projecting the specimen absorption. Therefore, computed tomography based on a tilt series of X-ray microscopic images can be used to reconstruct the local linear absorption coefficient and image the three-dimensional specimen structure. To preserve the structural integrity of biological objects during image acquisition, microscopy is performed at cryogenic temperatures.

Tomography based on X-ray microscopic images was applied to study the distribution of male specific lethal 1 (MSL-1), a nuclear protein involved in dosage compensation in Drosphila melanogaster, which ensures that males with single $\mathrm{X}$ chromosome have the same amount of most X-linked gene products as females with two $\mathrm{X}$ chromosomes. Tomographic reconstructions of X-ray microscopic images were used to compute the local three-dimensional linear absorption coefficient revealing the arrangement of internal structures of Drosophila melanogaster cells. Combined with labelling techniques, nanotomography is a new technique to study the $3 \mathrm{D}$ distribution of selected proteins inside whole cells.

We want to improve this technique with respect to resolution and specimen preparation. The resolution in the reconstruction can be significantly improved by reducing the angular step size to collect more viewing angles, which requires an automated data aquisition. In addition, fast-freezing with liquid ethane instead of cryogenic He gas will be applied to improve the vitrification of the hydrated samples. We also plan to apply cryo X-ray nanotomography in order to study different types of cells and their nuclear protein distributions.

\section{Acknowledgements}

We wish to thank D. Attwood and G. Schmahl for supporting this work, G. Denbeaux for his help with XM-1, B. Bates for the excellent workshop support and B. Harteneck for electroplating the zone plate objective. This work is funded partly by the Deutsche Forschungsgemeinschaft under contract SCHN 529/1-1 and the U.S. Department of energy, office of Basic Energy Science.

\section{List of References}

1. G. Schneider, Ultramicroscopy 75, 85 (1998).

2. S. Vogt, G. Schneider, A. Steuernagel, J. Lucchesi, E. Schulze, D. Rudolph, and G. Schmahl, Journal of Structural Biology 132, 123 (2000).

3. W. Meyer-Ilse, D. Hamamoto, A. Nair, S. A. Lelièvre, G. Denbeaux, L. Johnson, A. L. Pearson, D. Yager, M. A. Legros and C. A. Larabell, Journal of Microscopy 201(3), 395 (2001).

4. W. S. Haddad, J. E. Trebes, E. H. Anderson, R. A. Levesque \& L. Yang, Science 266, 1213 (1994).

5. J. Lehr, Optik 104, 166 (1997).

6. D. Weiß, G. Schneider, B. Niemann, P. Guttmann, D. Rudolph and G. Schmahl, Ultramicroscopy 84, 185 (2000).

7. E.H. Anderson et al., J. Vac. Sci. Technol. B18(6), 2970 (2000).

8. H.H. Hopkins and P.M. Barham, Proc. Phys. Soc. 63, 72 (1950).

9. P. Penczek, M. Marko, K. Buttle, and J. Frank, Ultramicroscopy 60, 393 (1995). 